\title{
Síndrome de Jael - Um relato de caso
}

\author{
Jael's Syndrome - A case report \\ Síndrome de Jael - Reporte de un caso
}

João Francisco Barbosa Cordeiro

ORCID: https://orcid.org/0000-0001-9977-6391

Universidade do Oeste de Santa Catarina, Brasil

E-mail: jfcbarbosa16@gmail.com

Mateus Diego Pavelski

ORCID: https://orcid.org/0000-0002-1691-390X Universidade Estadual Paulista Júlio de Mesquita Filho, Brasil E-mail: mateus_pavelski@hotmail.com

Aline Alves Luciano

ORCID: https://orcid.org/0000-0002-8114-2048 Clínica Privada, Brasil

E-mail: aline.alvesluciano@hotmail.com Osvaldo Magro Filho ORCID: https://orcid.org/0000-0002-9821-2479 Universidade Estadual Paulista Júlio de Mesquita Filho, Brasil E-mail: Osvaldo.magro@unesp.br

Tharzon Barbieri

ORCID: https://orcid.org/0000-0002-3346-152X Universidade do Oeste de Santa Catarina, Brasil E-mail: tharzon.barbieri@unoesc.edu.br

Maicon Douglas Pavelski

ORCID: https://orcid.org/0000-0002-9743-3034

Universidade do Oeste de Santa Catarina, Brasil

E-mail: maiconpavelski@hotmail.com

\begin{abstract}
Resumo
Os traumas frequentemente envolvem a área maxilofacial, porém os ferimentos de arma branca em face são raros. O mecanismo protetor de defesa ativa instintivamente defendendo a face de quaisquer lesões possíveis. Devido a essa incidência menor e também as diversas formas de ferimentos e objetos utilizados para tal injúria o ferimento por arma branca se torna um desafio aos cirurgiões. O cirurgião deve estar completamente familiarizado e preparado para atender essa demanda no pronto socorro. O objetivo do presente trabalho é relatar um caso de agressão com arma branca em face. O trauma gerado por um membro de sua família contra a mulher ocasionou um ferimento em região infraorbitária esquerda, transfixando até a região do palato onde era possível visualizar a outra extremidade da faca. Foi realizado procedimento cirúrgico sob anestesia geral para remoção do corpo estranho e síntese dos tecidos. A paciente se recuperou bem sem nenhuma complicação mais grave, somente com parestesia do nervo infraorbitário esquerdo. A paciente permaneceu em acompanhamento por dois meses e não compareceu as outras consultas pós-operatórias mais longas para uma avaliação a longo prazo.Incluir o resumo.
\end{abstract}

Palavras-chave: Traumatismos maxilofaciais; Ferimentos perfurantes; Violência contra a mulher.

\begin{abstract}
The traumas often involve the maxillofacial area, but stab wounds to the face are rare. The protective defense mechanism activates instinctively defending the face from any possible injuries. Due this lower incidence and also the various forms of injuries and objects used for such injury, stab wounds become a challenge for surgeons. The surgeon must be completely familiar and prepared to meet this demand on the emergency room. The purpose of this paper is to report a case of aggression with bladed weapon. The trauma generated by a member of his family against a woman caused an injury in the left infraorbital region, transfixing until the region of hard palate, where it was possible to see the end of the knife. A surgical procedure was performed under general anesthesia to remove the foreign body and synthesize the tissues. The patient recovered well, without any more serious complications, only with paraesthesia of the left infraorbital nerve. The patient remained in follow-up for two months and did not attend the other appointments for a long-term evaluation.
\end{abstract}

Keywords: Maxillofacial injuries; Wounds stab; Violence against women.

\section{Resumen}

El trauma a menudo afecta el área maxilofacial, pero las heridas por arma blanca en la cara son raras. El mecanismo de defensa protectora se activa instintivamente defendiendo el rostro de posibles lesiones. Debido a esta menor incidencia 
y también a las diversas formas de lesiones y objetos utilizados para tal lesión, las heridas por arma blanca se convierten en un desafío para los cirujanos. El cirujano debe estar completamente familiarizado y preparado para satisfacer esta demanda en la sala de emergencias. El propósito de este artículo es reportar un caso de agresión con arma blanca. El trauma generado por un familiar contra la mujer provocó una herida en la región infraorbitaria izquierda, traspasándola a la región del paladar donde se podía ver el otro extremo del cuchillo. Se realizó un procedimiento quirúrgico bajo anestesia general para remover el cuerpo extraño y sintetizar los tejidos. La paciente se recuperó bien sin mayores complicaciones, solo con parestesia del nervio infraorbitario izquierdo. El paciente permaneció en seguimiento durante dos meses y no acudió a las otras visitas postoperatorias más largas para una evaluación a largo plazo.

Palabras clave: Traumatismos maxilofaciales; Heridas punzantes; Violencia contra la mujer.

\section{Introdução}

O trauma pode ser visto como o conjunto das desordens causadas abruptamente por um agente etiológico físico, podendo apresentar-se em qualquer segmento corpóreo. Segundo dados da Organização Mundial da Saúde, este conjunto está entre as principais causas de morte e morbidade no mundo, estimando-se que aproximadamente 1,24 milhões de pessoas perdem a vida em consequência do trauma maxilofacial, sendo que as lesões da cabeça e da face podem equivaler a 50\% de todos os óbitos traumáticos (Moura et al., 2017).

A região maxilofacial é frequentemente acometida por lesões causadas por acidentes domésticos, trabalhistas, esportivos ou de trânsito, além de ferimentos por arma branca ou de fogo e agressão física. As lesões faciais variam em sua complexidade e apresentação, sendo tratadas conforme sua extensão, nível de contaminação, profundidade e do agente etiológico, necessitando uma abordagem especial objetivada em reestabelecer a forma e função estética e fisiológica do paciente. Para que essa abordagem seja realizada da melhor maneira possível, faz-se necessário que o Cirurgião Bucomaxilofacial possua o conhecimento dos princípios básicos de tratamento dessas injúrias, como os tipos de lesão em tecido mole da face, tempo de espera para a intervenção e a manipulação correta dos tecidos (Paiva et al., 2013; Provasi et al., 2018).

A incidência de trauma facial teve um aumento significativo nos últimos anos, sendo o motivo de queda a principal causa. Contudo, profissionais da saúde frequentemente não conseguem precisamente identificar a etiologia da lesão, muitas vezes por omissão da vítima. Tratando-se da violência contra a mulher, uma dúvida surge: os fatores relatados que originaram o trauma realmente representam o ocorrido ou há a possibilidade da omissão de casos de violência praticados no ambiente doméstico. A omissão de informações verídicas e a recusa em denunciar práticas violentas resultam em uma maior exposição aos fatores de risco e a limitação das ações dos profissionais da saúde durante o manejo da vítima (Ramos et al., 2018).

As lesões traumáticas provocadas por arma branca em região maxilofacial são pouco relatadas na literatura. A nomenclatura "Síndrome de Jael" foi empregada para determinar o trauma crânio-facial ocasionado intencionalmente por faca fazendo referência à história bíblica onde Jael assassina Sísera (Almeida Júnior et al., 2010).

Embora seja tratada como uma região predominante nos casos de lesões traumáticas, os ferimentos penetrantes por armas brancas são raros na área maxilofacial devido a tentativa de autodefesa fazendo uso das mãos para proteção. Em razão disso, o manejo de pacientes vítimas desse tipo de lesão em face apresenta um atendimento pouco padronizado em virtude da ausência de protocolos estabelecidos para o manejo da vítima (Dominguete et al., 2013; Moura et al., 2017).

De modo geral, os objetos penetrantes utilizados em agressões são rígidos o suficiente para transpassar diferentes estruturas anatômicas. Com isso, além do risco ocasionado pelo ataque violento, a remoção de tais instrumentos também caracteriza um potencial risco, requerendo uma avaliação criteriosa das estruturas envolvidas, principalmente os vasos sanguíneos (Nogueira Neto et al., 2015). Visando obter resultados satisfatórios no tratamento das lesões provocadas por arma branca, o conhecimento da anatomia e dos princípios básicos de cirurgia são extremamente importantes (Chowdhury et al., 2016; Provasi et al., 2018).

O tratamento das lesões faciais inclui a síntese dos tecidos moles através de suturas, redução e fixação de fraturas ósseas ou ainda, em casos de perda de substância, reconstruções teciduais, sejam eles duros ou moles (Paiva et al., 2013). A penetração 
do corpo estranho no momento da ferida pode causar infecção, principalmente objetos que formam uma cavidade. Durante o ato cirúrgico, o debridamento da ferida reduz o potencial de infecção, contudo, a administração de antibióticos no transoperatório e a profilaxia antitetânica são seguramente indicados (Petersen \& Waterman, 2011).

Deste modo, o presente trabalho tem como objetivo relatar um caso de ferimento por arma branca em região infraorbitária esquerda de face, ocasionada pela violência interpessoal entre familiares de uma mesma casa (Paiva et al., 2013).

\section{Metodologia}

Trata-se de um Relato de Caso Clínico, de um paciente que realizou o tratamento durante seu internamento hospitalar (Lüdke \& André, 1986; Koche, 2011). Os dados epidemiológicos, dados médicos e registros fotográficos, foram coletados através do prontuário físico e eletrônico, após a permissão da paciente e a assinatura do Termo de Consentimento Livre e Esclarecido (TCLE), seguido as recomendações de ética da Declaração de Helsinque (1984) e também seguido os preceitos da publicação sem a identificação do paciente.

\section{Relato de Caso}

O paciente do gênero feminino, 42 anos de idade, sem comorbidades associadas, sem medicação de uso contínuo e sem alergias, deu entrada na emergência do Hospital, com histórico de agressão física por sua enteada. A agressão física foi realizada com uma arma branca (faca) inserida em face, na região infraorbitária esquerda com transfixação do objeto para a cavidade bucal em região de palato (Figura 1).

A paciente deu entrada no pronto-socorro consciente, orientada, Glasgow 15, vias aéreas pérvias, sem alterações oculares ou visuais, com quadro de epistaxe, parestesia infraorbitária esquerda e com a arma branca inserida na face.

Após avaliação médica inicial e com sinais vitais estáveis a paciente foi encaminhada para avaliação da equipe de cirurgia e traumatologia bucomaxilofacial. Durante o exame físico observou-se a faca introduzida na região infraorbitária esquerda com uma extremidade visível, sem sinais de comprometimento do globo ocular e ausência de oftalmoplegia, distopia e diplopia. 
Research, Society and Development, v. 10, n. 2, e32510212688, 2021

(CC BY 4.0) | ISSN 2525-3409 | DOI: http://dx.doi.org/10.33448/rsd-v10i2.12688

Figura 1. Aspecto inicial da paciente, já em centro cirúrgico sob anestesia geral.

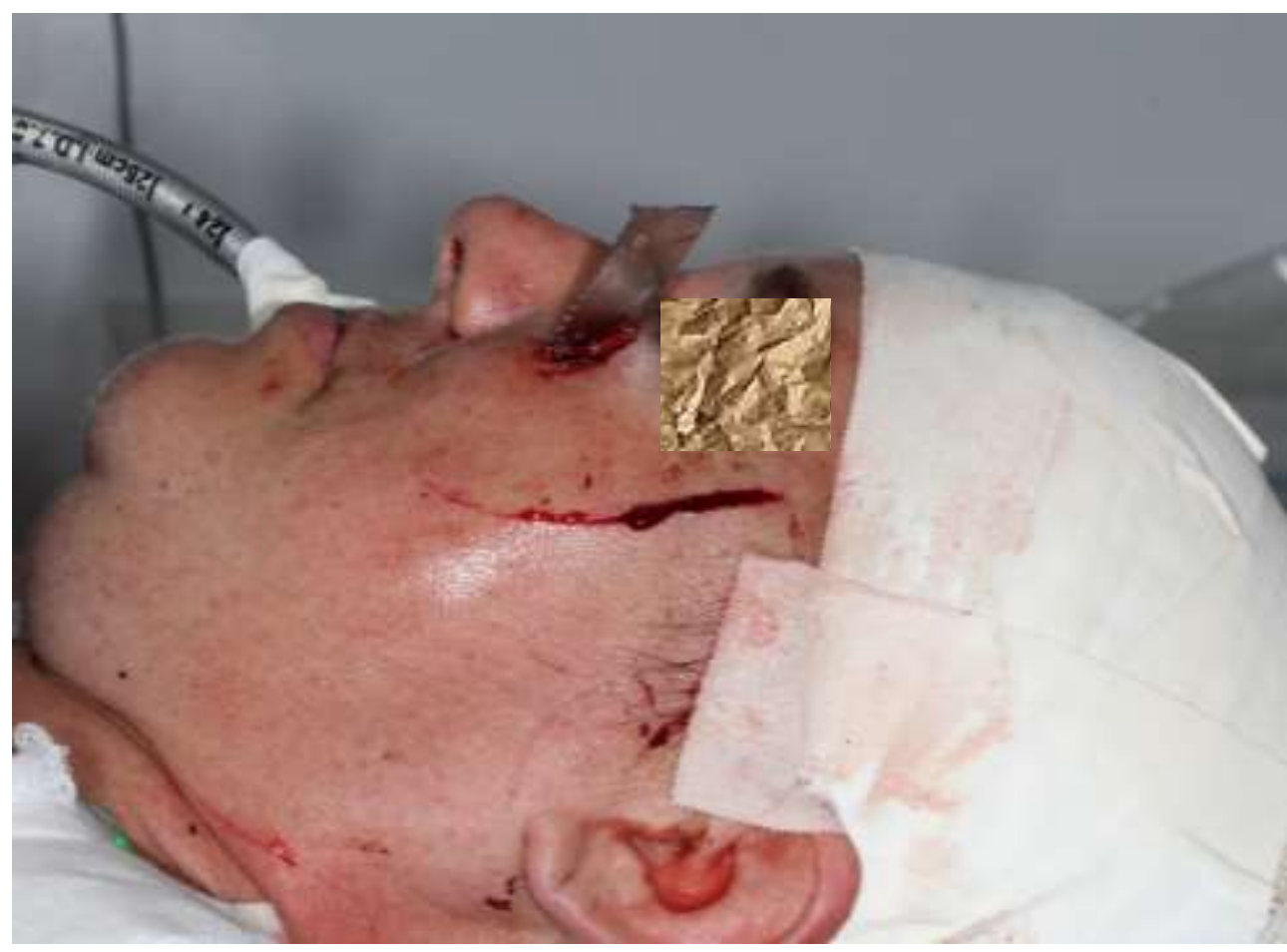

Fonte: Autores.

Nota-se o direcionamento latero-medial da faca diretamente abaixo do globo ocular, sem afetar o globo ocular e a pálpebra inferior, adentrando a face em um sentido súpero-inferior.

O ferimento era transfixante da região infraorbitária, adentrando o seio maxilar, cavidade nasal e ultrapassando para a cavidade bucal, sendo observada a outra extremidade da faca no palato à direita (Figura 2).

Figura 2. Aspecto intraoral da paciente, com a extremidade da faca visível.

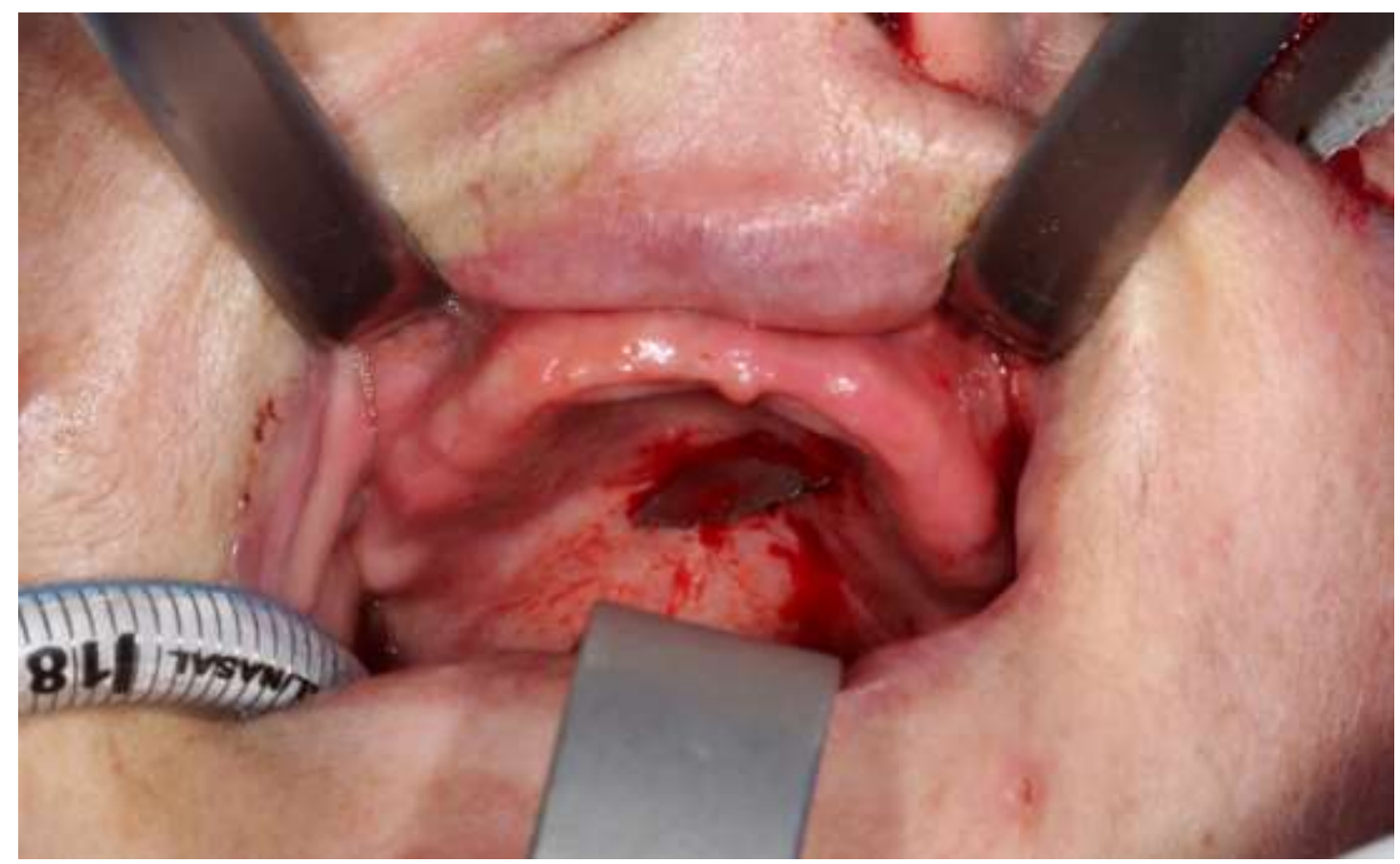

Fonte: Autores. 
Foram realizados exames complementares de coagulação sanguínea e tomografia computadorizada de face onde verificou-se que o trajeto exercido pela faca transpassava a parede anterior do seio maxilar esquerdo, a cavidade nasal, o terço inferior do septo nasal cartilaginoso e palato duro direito chegando a cavidade bucal.

Figura 3. Tomografia computadorizada de face, na reconstrução 3D.

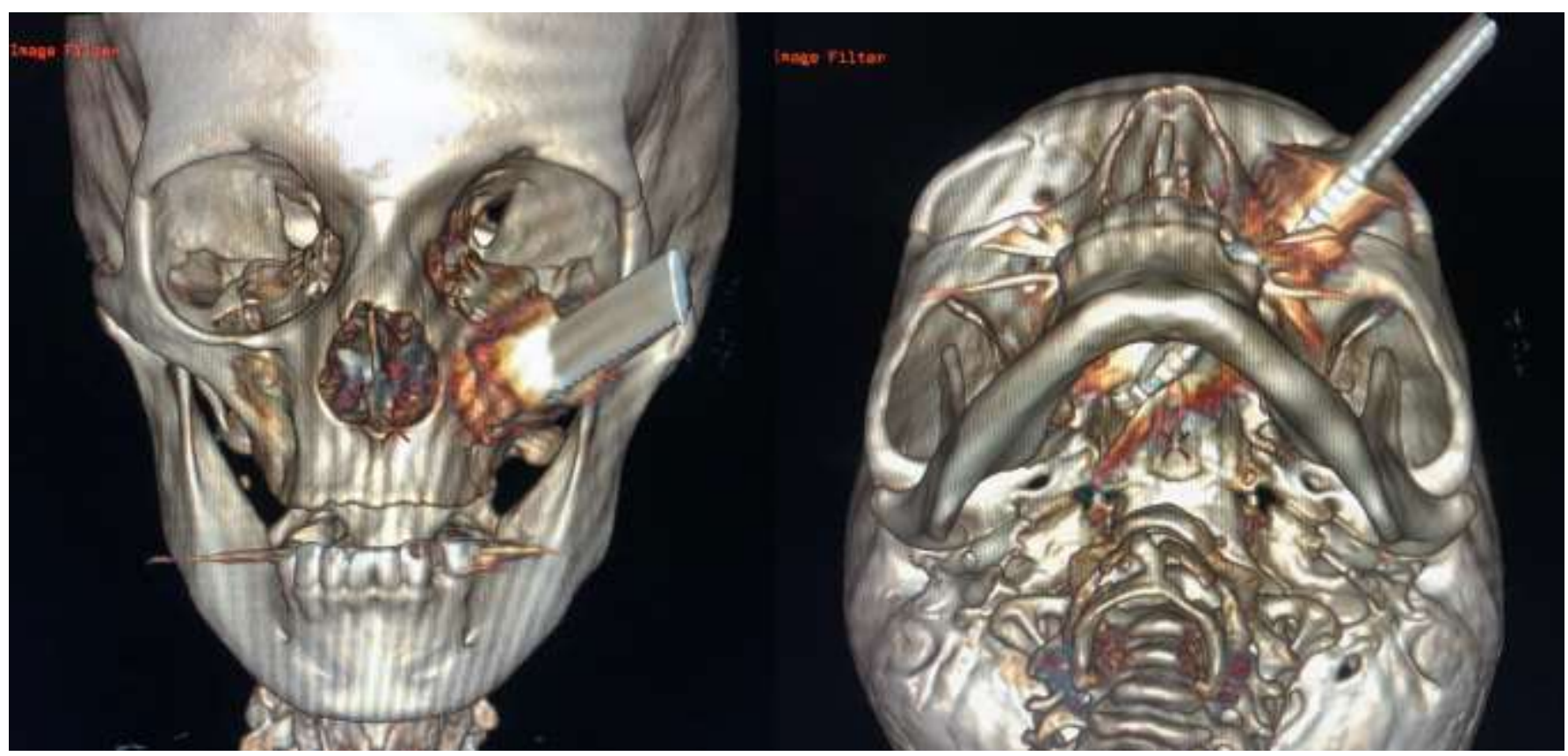

Fonte: Autores.

A paciente foi então conduzida ao centro cirúrgico onde, sob anestesia geral foi realizada a remoção do corpo estranho, obtendo-se a remoção total da arma branca (faca) em um único corpo, sendo realizada a limpeza do ferimento, a hemostasia e a sutura por planos de todos ferimentos cortantes. Não houve sangramento anormal na região do trauma e o procedimento não apresentou intercorrências durante o ato cirúrgico. A paciente evoluiu bem e no dia seguinte a intervenção cirúrgica foi liberada do hospital. 
Figura 4. Aspecto extra-oral da paciente em pós-operatório de 15 dias.

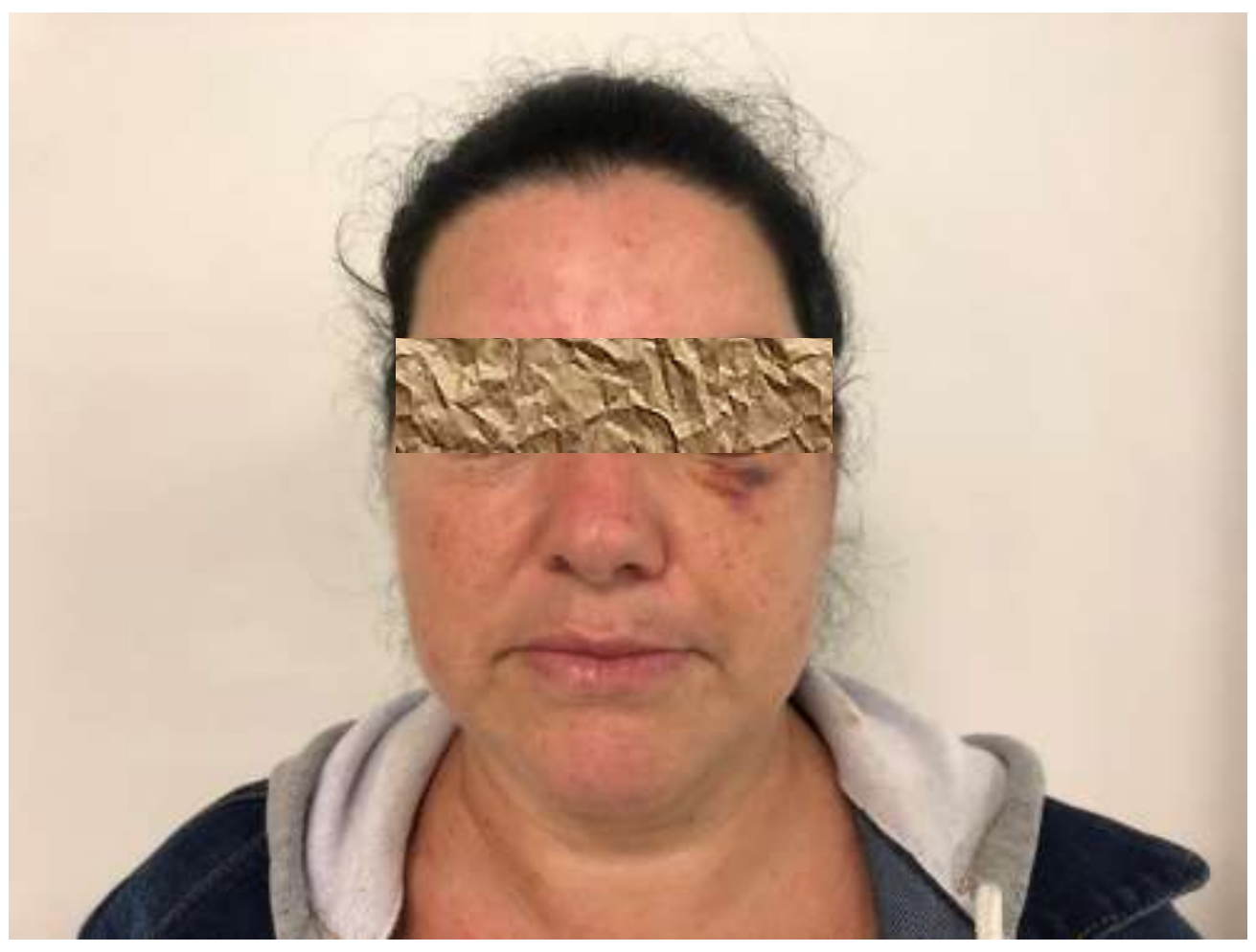

Fonte: Autores.

Note a boa cicatrização da face da paciente, sem sinais de cicatriz hipertrófica e ferimentos com ferimentos discretos após quinze dias de pós-operatório. A paciente se queixava somente da parestesia da região infra-orbitária.

\section{Resultados e Discussão}

A paciente permaneceu em acompanhamento ambulatorial por três meses evoluindo com parestesia do nervo infraorbitário esquerdo, sem outras alterações. Não foi possível realizar um acompanhamento a longo prazo pois a paciente não compareceu aos retornos agendados pela equipe após esta data e não foi possível contactar a paciente.

De acordo com o estudo epidemiológico do trauma bucomaxilofacial por Ramos et al., o segundo fator etiológico mais frequente para os traumas maxilofaciais foi a agressão física, ficando atrás apenas dos acidentes com motocicleta, incidentes em ambos os sexos.

Os traumas perfurantes diferem-se dos traumas contundentes pois a lesão traumática é resultado de objetos pontiagudos que transpassam a pele, sendo classificados de acordo com sua energia. Desta forma, a velocidade que o objeto penetra tem mais importância quando comparado ao seu tamanho e sua forma para a formação do trauma. Assim, as lesões perfurantes podem ser dividas em energia Muito Baixa, como ferimentos por faca, Baixa Energia, como projéteis de revolveres, e Alta Energia, como projéteis de rifles (Petersen \& Waterman, 2011).

Em um estudo transversal de três hospitais, SILVA et al. verificaram registros de 7063 vítimas de trauma maxilofacial. Destes, 55,1\% decorrentes de violência interpessoal e 44,9\% de acidentes com automóveis ou motocicletas. A maior parte das vítimas era do gênero masculino (71,2\%) apresentando uma média de idade de 29,6 anos, sendo que a maioria das vítimas possuía idade entre 30 e 50 anos. Acontecendo majoritariamente durante os dias úteis da semana (60,07\%), no período noturno (59,52\%), observou-se prevalência da agressão nua $(47,85 \%)$ resultando em lesões em tecidos moles $(43,21 \%)$ e fraturas simples $(31,63 \%)$.

Atualmente, a violência contra a mulher constitui um amplo problema social e de saúde pública, caracterizando um 
fenômeno ímpar quanto a classe social, raça/etnia, idade e grau de escolaridade das vítimas. Livre do status da mulher, o locus dessa barbárie permanece no âmbito familiar.

A literatura evidencia a dificuldade no estabelecimento da relação entre o trauma maxilofacial e a violência doméstica, pelo fato da frequente omissão da mulher sobre a verdadeira etiologia das agressões. Ressalta-se também que, em geral as vítimas encontram-se em estado emocional bastante fragilizado, omitindo informações por vergonha, visto que em alguns casos a justificativa para a agressão pode ter sido instigada pelo uso de substâncias ilícitas ou bebidas alcoólicas (Almeida Júnior et al., 2010; Ramos et al., 2018).

Segundo Almeida Júnior et al., uma revisão dos aspectos socioeconômicos de 254 vítimas de trauma por arma branca e de fogo, as características predominantes foram: homens melanodermas, idade entre 15 e 35 anos, usuários de drogas lícitas e ilícitas e de baixo nível socioeconômico. Os incidentes regularmente são registrados na sexta-feira ou sábado, entre as 21 e $02 \mathrm{~h}$, induzidos por brigas caseiras. Apesar da violência desse caso ser realizada por um membro da família a paciente relatou precisamente a etiologia, negou o envolvimento de bebidas alcoólicas ou substâncias ilícitas como justificativa e identificou o membro familiar agressor como sua enteada, não se tratando, portanto, de um agressor masculino, o que difere da maioria dos relatos da literatura (Costa et al., 2014).

No caso em questão, estando em partes de acordo com a literatura, a paciente é do sexo feminino, 42 anos de idade, de baixo nível socioeconômico, porém, o trauma acontecido em uma quinta-feira (Almeida Júnior et al., 2010; Ramos et al., 2018).

O paciente acometido por trauma facial deve ser avaliado sistematicamente, a fim de identificar lesões em estruturas anatômicas importantes como o nervo facial, glândula parótida e o ducto parotídeo. A presença de sangramento ativo na área lesionada, hematoma crescente, baixo nível de hemoglobina e sinais hipovolemia são indicativos de lesão vascular associada (Almeida Júnior et al., 2010). No presente relato, os exames não apontaram nenhuma alteração significativa, e o corpo estranho felizmente não atingiu nenhuma outra estrutura nobre ou vascular de grande calibre, o que poderia dificultar o seu manejo e causar grandes danos a qualidade de vida da paciente

A disposição do instrumento na face denotava que a agressão ocorreu no sentido ântero-posterior e de cima para baixo em região infraorbitária esquerda, atingindo o feixe vásculo-nervoso infraorbitário, próximo a outras estruturas nobres, que felizmente não foram atingidas. Também foi possível identificar a mão dominante direita da agressora, visto que o objeto foi inserido em um trajeto látero-medial tendo seu ponto de entrada em região facial esquerda e cruzando a direita à medida em que o objeto foi inserido.

A pele e as mucosas da região maxilofacial são ricas em microorganismos, mas não possuem grandes massas musculares. Com isso, o risco de cavitação, necrose e infecção subsequente é menor quando comparado ao trauma perfurante em outras regiões (Petersen \& Waterman, 2011).

Para o trauma maxilofacial de energia muito baixa por via oral, mucosa e pele facial, a utilização de antibióticos demonstram uma redução de quadros infecciosos de $27 \%$ a $7 \%$ administrando penicilinas ou cefalosporinas no tran soperatório (Petersen \& Waterman, 2011).

O manejo do trauma facial caracteriza-se por minimizar cicatrizes e evitar mais lesões em estruturas adjacentes. A estrutura óssea é responsável pela proteção de diversos componentes anatômicos importantes, sendo imperativo que estes sejam verificados no momento da avaliação inicial e dependendo da lesão dos tecidos moles da lesão primária, as localizações das fraturas não podem ser corrigidas em um primeiro momento. As lacerações devem ser irrigadas abundantemente, debridadas e, preferencialmente, suturadas por planos (Morris \& Kellman, 2013).

Dentre as complicações pré-operatórias mais frequentes do trauma por arma branca pode-se citar deformidade facial, injúria orbital, maloclusão, anosmia e telecanto, enquanto que comumente no pós-operatório a parestesia e paralisia, a diplopia, a deformação facial e a sinusite crônica, são os mais recorrentes (Affonso et al., 2010; El Sayed et al., 2018; Silva et al., 2014). 


\section{Considerações Finais}

As lesões causadas pelas agressões por arma branca não expressam um padrão definido, mas apresentam um perfil de agressor majoritário e que recebe uma atenção especializada nas políticas públicas de saúde, mas que ainda se fazem presentes atualmente nos centros de trauma. O cirurgião-dentista bucomaxilofacial deve estar preparado para manejar estes casos desafiadores e devem individualizar o seu manejo de acordo com a necessidade de cada caso.

\section{Referências}

Affonso, P. R. A., Cavalcanti, M. A., Groisman, S., \& Gandelman, Í. (2010). Etiologia de trauma e lesões faciais no atendimento pré-hospitalar no Rio de Janeiro. Artigos Originais, 1-9.

Almeida Júnior, P., Santos, T. de S., Kumar, P. N., Martins Filho, P. R. S., \& Carvalho, R. W. F. de. (2010). Ferimento a faca impactada na face (Síndrome de Jael): relato de caso. Rev. Cir. Traumatol. Buco-Maxilo-Fac, 5458, 9-13.

Chowdhury, F. H., Haque, M. R., Hossain, Z., Chowdhury, N. K., Alam, S. M., \& Sarker, M. H. (2016). Nonmissile Penetrating Injury to the Head: Experience with 17 Cases. World Neurosurgery, 94, 529-543. https://doi.org/10.1016/j.wneu.2016.06.062.

Costa, M. C. F., Cavalcante, G. M. S., Nóbrega, L. M. da, Oliveira, P. A. P., Cavalcante, J. R., \& d'Avila, S. (2014). Facial traumas among females through violent and non-violent mechanisms. Brazilian Journal of Otorhinolaryngology, 80(3), 196-201. https://doi.org/10.1016/j.bjorl.2013.10.001.

Dominguete, P. R., Matos, B. F., Meyer, T. N., \& Oliveira, L. R. (2013). Jael syndrome: Removal of a knife blade impacted in the maxillofacial region under local anaesthesia. BMJ Case Reports, April. https://doi.org/10.1136/bcr-2013-008839.

El Sayed, M., Hassan Saad, R., \& Fereir, A. (2018). Undiagnosed impacted knife blade from a penetrative orbital injury: A case report. International Journal of Surgery Case Reports, 53, 254-258. https://doi.org/10.1016/j.ijscr.2018.10.064.

Koche, J. C. (2011). Fundamentos de metodologia científica. Vozes.

Lüdke, M., \& André, M. (1986). Pesquisa em educação: abordagens qualitativas. E.P.U.

Morris, L. M., \& Kellman, R. M. (2013). Complications in Facial Trauma. Facial Plastic Surgery Clinics of North America, 21(4), 605-617. https://doi.org/10.1016/j.fsc.2013.07.005.

Moura, M. T. F. L. de, Daltro, R. M., \& Almeida, T. F. de. (2017). Traumas faciais: uma revisão sistemática da literatura. Revista Da Faculdade de Odontologia - UPF, 21(3), 331-337. https://doi.org/10.5335/rfo.v21i3.6158.

Nogueira Neto, J., Muniz, V., Figueiredo, L., Freire, F., \& Souza, A. (2015). Ferimento provocado por arma branca impactada em região maxilofacial: Relato de caso. Revista de Cirurgia e Traumatologia Buco-Maxilo-Facial, 15(1), 41-44.

Paiva, L. G. J., Rodrigues, Á. R., Carneiro, R. P., Oliveira, M. T. F., Silva, M. C. P., \& Barbosa, D. Z. (2013). Fratura mandibular após ferimento por arma branca - diagnóstico e conduta clínica Mandibular fracture after stab wounds - diagnosis and clinical conduct. Rev Odontol Bras Central, 21(61), 100-102.

Petersen, K., \& Waterman, P. (2011). Prophylaxis and treatment of infections associated with penetrating traumatic injury. Expert Review of Anti-Infective Therapy, 9(1), 81-96. https://doi.org/10.1586/eri.10.155.

Provasi, S., Geraldo, A. H. P. da S., Oku, A. T., \& Paulesini Junior, W. (2018). Trauma facial: ferimento por arma branca. Relato de caso. Revista de Odontologia Da Universidade Cidade de São Paulo, 29(3), 305. https://doi.org/10.26843/ro_unicidv2932017p305-311.

Ramos, J. C., de Almeida, M. L. D., de Alencar, Y. C. G., Filho, L. F. de S., Figueiredo, C. H. M. da C., \& Almeida, M. S. C. (2018). Epidemiological study of bucomaxilofacial trauma in a Paraíba reference hospital. Revista Do Colegio Brasileiro de Cirurgioes, 45(6), 1-9. https://doi.org/10.1590/0100-6991e-20181978.

Silva, C. J. de P., Ferreira, R. C., de Paula, L. P. P., Haddad, J. P. A., Moura, A. C. M., Naves, M. D., \& e Ferreira, E. F. (2014). Traumatismos maxilofaciais como marcadores de violência urbana: Uma análise comparativa entre gêneros. Ciencia e Saude Coletiva, 19(1), 127-136. https://doi.org/10.1590/141381232014191.2059 . 